\title{
Cervical ectopic pregnancy misdiagnosed as an incomplete miscarriage - a steep learning curve
}

\author{
Harjit S Dhaliwal, Jasmina Glogic, Azal El Adwan, Rachna Dwivedi
}

\begin{abstract}
Corresponding author: Dr Harjit S Dhaliwal, Royal Bournemouth Hospital, Department of Obstetrics and Gynaecology, Bournemouth, UK; Email - harjitdhaliwal2004@yahoo.co.uk
\end{abstract}

Distributed under Attribution-Non Commercial - Share Alike 4.0 International (CC BY-NC-SA 4.0)

\begin{abstract}
Cervical pregnancy is a rare form of an ectopic pregnancy that accounts for $<1 \%$ of all ectopic pregnancies. It results from implantation of the blastocyst in the endocervical canal below the level of the internal os. In the UK the approximate incidence of ectopic pregnancies is 11/1000 with an estimate of around 11,000 ectopic pregnancies diagnosed annually. Although non-tubal ectopic pregnancies account for only 5\%of ectopic pregnancies, they contribute to a significant morbidity. Management options for cervical ectopic pregnancies range from conservative drug treatment to radical surgical procedures. Over, the last few decades, the mortality rates of ectopic pregnancies have been reduced. This is due to the early recognition of the condition, improvement in laboratory assay sensitivities, dedicated EPAU (Early pregnancy assessment units), ultrasound technology and finally minimally invasive surgical procedures. We present a case of a 38 year old who was 6 weeks pregnant in her first pregnancy. She initially presented with heavy vaginal bleeding followed by cramping lower abdominal pain. Her initial scan was inconclusive. A follow up scan a week later suggested an incomplete miscarriage. A high clinical index of suspicion was based on the rising serum beta $\mathrm{HCG}$ and the findings in theatre.
\end{abstract}

Keywords: Cervical ectopic pregnancy, ultrasound diagnosis.

Cervical ectopic pregnancy is an uncommon clinical entity in which the pregnancy implants into the cervical mucosa. It has a varying incidence of $1: 1000$ to $1: 95,000$ pregnancies ${ }^{1}$. The aetiology is still unclear. Various theories have been proposed: a) Rapid transport of the fertilised ovum to the cervical canal before it is capable of nidation or an unreceptive endometrium. b) Damage to the cervix and endometrial lining during operative procedures. The most common being dilatation and curettage followed by previous caesarean delivery. Other risk factors are intrauterine devices, previous surgical termination of pregnancy, anomalies of the reproductive tract and Asherman's syndrome. ${ }^{2}$ The prevalence of cervical ectopic is increasingly being reported with assisted reproductive techniques including both intrauterine and intrafallopian tube embryo transfers $^{3}$. One should realise that due to the rarity, the absolute risk remains small and the association with the above factors remain weak.

\section{Case}

A 38 years old was referred by her general practitioner to our early pregnancy assessment unit with heavy vaginal bleeding associated with clots and cramping lower abdominal pain of one-day duration. The intensity of the bleeding and pain has slowed down on presentation to the unit the following morning. She was in her first pregnancy and was approximately in 6 weeks of gestation. Her

Received: $28^{\text {th }}$ February 2020. Accepted: $29^{\text {th }}$ April 2020.

Dhaliwal HS, Glogic J, El Adwan A, Dwivedi R. Cervical ectopic pregnancy misdiagnosed as an incomplete miscarriage - a steep learning curve. The New Indian Journal of OBGYN. 2020; 7(1): 111-4. 
menstrual cycles had been regular. This was a planned natural conception. There were no attributing risk factors previous surgical termination of pregnancy, operative cervical or uterine procedures or insertion of intrauterine devices.

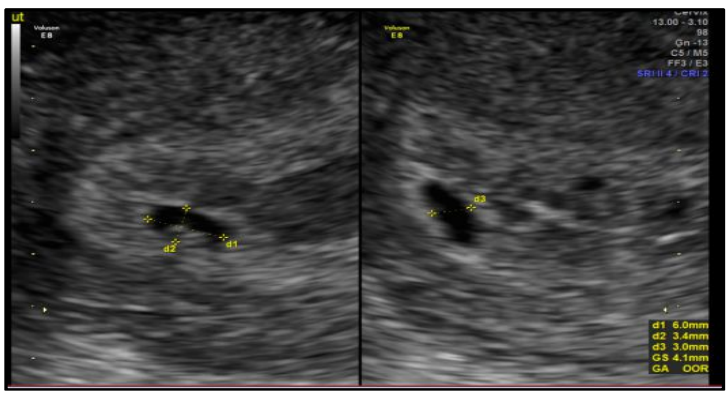

Figure 1: Cystic lesion in upper endometrium

Her initial scan on presentation suggested an endometrial thickness of $12 \mathrm{~mm}$ and a disrupted endometrial midline echo. The ovaries and the adnexa were normal. There were mixed echoes and fluid within the endometrial cavity and a collection of mixed solid echoes in the cervical canal measuring $31 \times 29 \mathrm{~mm}^{2}$. There was also a cystic structure in the upper endometrium (Figure - 1). Raising a possibility of an intrauterine gestation sac of $<5$ weeks gestation. Her initial beta $\mathrm{HCG}$ was $8083 \mathrm{IU} / \mathrm{ml}$. She was reviewed 48 hours later where her pains had subsided and only had mild vaginal bleeding. A repeat serum beta HCG suggested an $82 \%$ rise $(14741 \mathrm{IU} / \mathrm{ml})$. Scan images were reviewed and

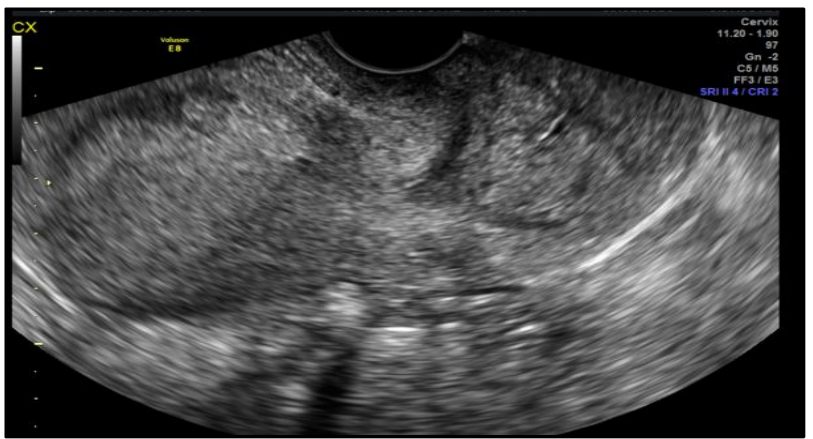

Figure 2: Heterogenous collection in cervical canal

was thought there was a likely intrauterine gestational sac. A further scan was organised a week from the original scan. Her bleeding and pains had settled few days prior to her appointment. A repeat scan suggested the absence of a gestation sac, normal ovaries with no evidence of masses, adnexal cysts and free fluid in the pouch of douglas. There was a heterogeneous collection measuring $32 \times 34 \times 13 \mathrm{~mm}^{3}$ in the cervical canal. The comment was that of an incomplete miscarriage (Figure - 2). A repeat beta HCG done suggested a rising $(26,370 \mathrm{IU} / \mathrm{ml})$ trend rather than a fall as would be expected from an incomplete miscarriage. The plan was for a clinical assessment and to consider a diagnostic laparoscopy and a suction evacuation of the uterine cavity.

On examination, the abdomen was soft, non-tender. Speculum examination suggested a cervical mass which was most likely a clot or products of conception. It was firmly attached to the cervical os. It was not possible to remove it with a polyp forceps and she started having a brisk bleed. She underwent fluid resuscitation and was immediately transferred to operation theatre for an examination under anaesthesia, evacuation of uterus and excision of cervical mass.

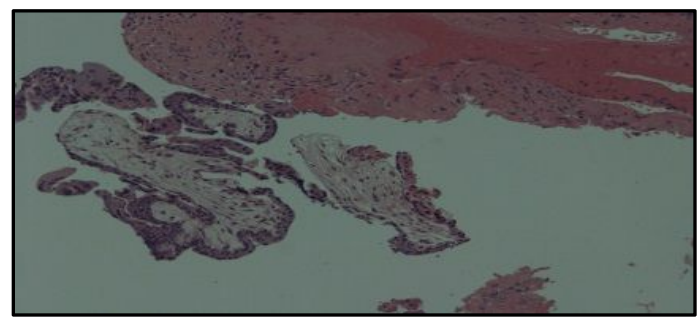

Figure 3: Chorionic villi.

The differentials were that of a probable cervical pregnancy, incomplete or inevitable miscarriage or a cervical malignancy. The spherical mass was about $3.5 \mathrm{cms}$ that was dilating the cervical canal. There was no clear plane between

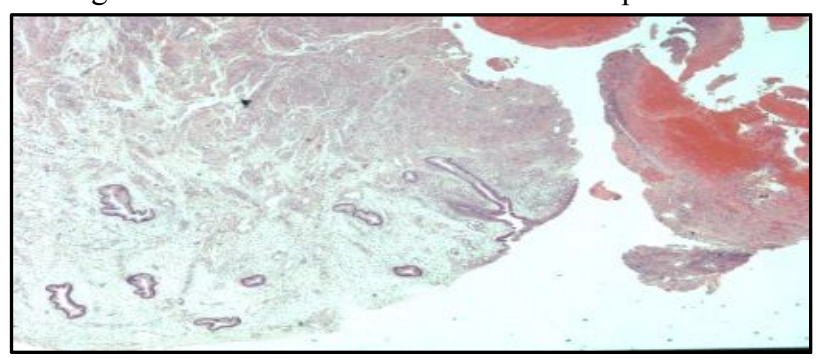

Figure 4: Cervical glands and trophoblastic tissue

mass and the cervix. It was contiguous with no obvious passage up the cervical canal. The margins were irregular and ill-defined. The mass was carefully resected and excised leaving a more normal appearance of the cervix. She then underwent an evacuation of the endometrial cavity. Ball diathermy was used at the site of the resected mass. A single dose of tranexamic acid was given intravenously. Complete haemostasis was achieved and her blood pressure had normalised. She received two units of blood transfusion followed by an iron transfusion. She was discharged home on day two. Prior to discharge her beta HCG levels had dropped to $3424 \mathrm{IU} / \mathrm{ml}$. Further drops were seen on day 7 and 14 (197 IU/ml, $15 \mathrm{IU} / \mathrm{ml})$. 


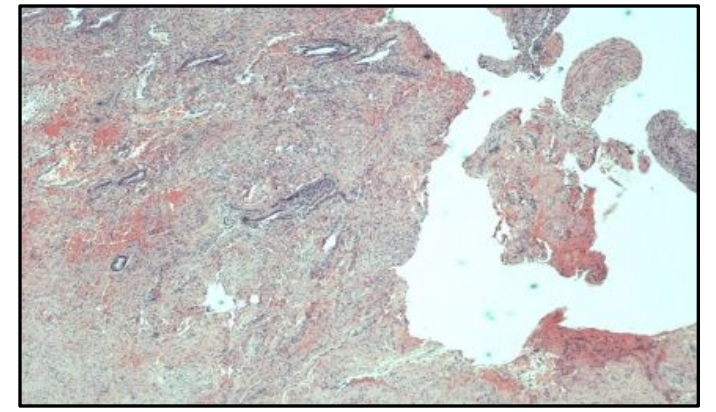

Figure 5: Implantation site.

Histopathology confirmed features consistent with a cervical pregnancy where cut sections showed endocervical tissue with implanted chorionic villi, trophoblastic tissue and decidua along with haemorrhage (trophoblastic tissue within the cervical stroma) - ectopic pregnancy that had implanted in the uterine endocervix (Figure - 3,4,5). The curetting's from the endometrial cavity suggested decidual tissue and endometrial glands with Arias Stella reaction. No chorionic villi or trophoblastic tissue was present.

\section{Discussion}

The diagnosis of early cervical ectopic pregnancy is possible with early transvaginal sonography and serum beta HCG assays. Early diagnosis is important as it essential for successful treatment and avoidance of acute potential complications like large volume of blood loss that might necessitate blood transfusions and hence emergency procedures such as a hysterectomy. It can have a detrimental impact to the patient both psychologically and physically. The main aim is to be able to distinguish between an early intrauterine pregnancy, an ongoing miscarriage where the gestational sac is passing through the cervix and a cervical pregnancy where the pregnancy is implanted within the cervical tissue. The presence of foetal cardiac activity helps to make a clear differentiation between a cervical ectopic and an ongoing miscarriage. In the latter, one should not see a foetal heart.

Cervical pregnancy was first described in 1817. In 1911, Rubin proposed the following histopathological criteria that is required in hysterectomy specimens ${ }^{4}-1$ ) cervical glands must be opposite the placental attachment, 2) placental attachment to the cervix must be situated below the entrance of the uterine vessels or below the reflection of the anterior and posterior surface of the uterus and finally foetal elements must be absent from the corpus uteri. Palman And McElin proposed a more practical criterion ${ }^{5}$. The 5 key points of the criteria are - 1) uterine bleeding which should be painless following a period of amenorrhoea, 2) a soft enlarged cervix equal to or larger than the corporal portion of the uterus - (an hourglass shaped uterus), 3) products of conception entirely in the endocervical canal and firmly attached to the endocervix, 4) a closed internal os and 5) a partially opened external os.

Diagnosis of a cervical pregnancy requires visualisation of the trophoblastic mass or intracervical ectopic gestational sac. The Ultrasound criteria for diagnosing a cervical pregnancy ${ }^{6}$ are closed internal os, gestational sac or placental tissue below the level of the uterine arteries, absent "Sliding sign" and high peritrophoblastic vascularity on doppler assessment. However Benson and Doubilet disputed the usefulness of this. Local endocervical tissue invasion is important in cervical pregnancy and may be possible to identify the site with the ultrasound. The cervical mucosa has no protection against the trophoblastic invasion and allows deep penetration of the proliferating chorionic villi into the fibromuscular layer. On ultrasound you will normally see a hyper echoic trophoblastic ring that will be thicker in the area of invasion.

Management options for a cervical ectopic range from drug therapies to more radical surgical procedures. Over the years there has been a significant shift in treatment modalities gearing towards a more conservative approach. Improved transvaginal ultrasound scanning facilities, dedicated assessment units and highly sensitive serum beta HCG assays have led to an improved understanding and detection at an earlier gestation and hence a more conservative management options can be implemented. Surgical treatment is usually reserved for patients with hemodynamic instability. Due to this rare clinical entity and lack of any retrospective studies, there are no clear guidelines for optimal treatment options for stable patients with a cervical pregnancy. Local injection of methotrexate or potassium chloride if a foetal heart is present via transvaginal or trans abdominal with or without systemic methotrexate have been advocated due the low risk of bleeding and preservation of future fertility. ${ }^{7}$ Some authors have also advocated a surgical curettage following a local injection of methotrexate is necessary as it will reduce a massive bleed after trophoblastic shedding from the atonic cervix that occurs from the metabolic effects of methotrexate. The success rate of administration has been reported to be in the region of $81 \%$, while the percentage increased to around $90 \%$ when combined with other conservative methods. Higher failure rate of a single methotraxate therapy is more likely if the gestational age is $>9$ weeks, beta HCG levels 
The New Indian Journal of OBGYN. 2020 (July-December); 7(1)

$>10,000 \mathrm{IU} / \mathrm{ml}$ or foetal $\mathrm{CRL}>10 \mathrm{~mm}$. Foeticide with potassium chloride would enhance the effectiveness of methotrexate. Uterine artery ligation, ${ }^{8}$ uterine embolization ${ }^{9}$, mechanical tamponade by Foley's and silicone double cervical ripening catheter have also been used as adjuncts in both medical and surgical management to treat or minimise the risk of bleeding leading to a reduction in hysterectomies performed.

Early cervical pregnancy can be mistaken for an incomplete or inevitable miscarriage as the products of conception is retained in the cervical canal by a resistant external cervical os thereby dilating the cervical canal as seen in our patient. Retrospect analysis of the still scan pictures suggests the findings as being misleading. There was a pseudosac in the endometrial cavity or the presence of a false gestational sac which was then not present on the follow up scan, closed internal os which was present on both the scans and finally a solid collection present on both the scans. Due to the rarity of this form of an ectopic pregnancy, a cervical pregnancy was not thought of and hence the sonographers vastly believed it to be an incomplete miscarriage. Rising serum beta HCG levels along with nonvisualisation of a gestational sac and a mass in the cervical canal both on the initial and subsequent scan should have alerted the attending clinician. She was seen in an outpatient setting and was examined where they thought it was products of conception in the cervical canal and decide to remove it. Being unable to remove it as being densely adhered to the cervical mucosa and the brisk bleeding prompted a change in diagnosis that was confirmed on histopathology. We the authors believe that the findings were more for a cervical pregnancy rather than that of a miscarriage.

\section{Conclusion}

Cervical ectopic pregnancy is a rare form of an ectopic pregnancy. Early diagnoses with the use of ultrasound and utilization of conservative drug therapies have led to a decrease in morbidity and mortality rates. Our case highlights the increased awareness to both the clinician and sonographers that the cervical site should always be kept in mind no matter how rare the incidence is.
Conflict of interest: None. Disclaimer: Nil.

\section{References}

1. Celik C, Baka, Acar A, Cezgine K, Akyurek C. Methotrexate for cervical pregnancy. A case report. J Reprod Med. 2003; 48:130-2.

2. Shinagawa S, Nagayama M. Cervical pregnancy as possible sequela of induced abortion. Report of 19 cases. Am J Obstet Gynecol. 1969; 105: 282-4.

3. WeyermanPC, Verhoeven AT, Alberda AT. Cervical pregnancy after in -vitro fertilization and embryo transfer. Am J Obstet Gynecol. 1989; 161:1145-7.

4. Rubin IC. Cervical pregnancy. Surg Gynecol Obstet. 1911; 13: 625.

5. Varghese U, Fajardo A, Gomathinayagam T. Cervical pregnancy. Oman Med J. 2008; 23:53-4.

6. Ushakov FB, Elchalal U, Aceman PJ, Schenker JG. Cervical pregnancy: Past and future. Ostet Gynecol Surv. 1997; 52: 45-9.

7. Doubilet PM, Benson CB, Frates MC, Ginsburg E. Sonographically guided minimally invasive treatment of unusual ectopic pregnancies. J Ultrasound Med. 2004; 23: $359-70$

8. Kung FT, Lin H, Hsu TY, Chang CY, Huang HW, Huang LY, et al. Differential diagnosis of suspected cervical pregnancy and conservative treatment with the combination of laparoscopy-assisted uterine artery ligation and hysteroscopic endocervical resection. Fertil Steril. 2004; 81:1642-9.

9. Zakaria MA, Abdallah ME, Shavell VI, Berman, JM, Diamond MP, Kmak DC. Conservative management of cervical ectopic pregnancy: utility of uterine artery embolization. Fertil Steril. 2011; 95:872-6.

\footnotetext{
Harjit S Dhaliwal ${ }^{1}$, Jasmina Glogic ${ }^{2}$, Azal El Adwan ${ }^{3}$, Rachna Dwivedi ${ }^{4}$

1,2,3,4 Royal Bournemouth Hospital, Department of Obstetrics and Gynaecology, Bournemouth, UK
} 